\title{
VALORES DE PRESSÃO ARTERIAL EM TRABALHADORES DE UMA INSTITUIÇÃO UNIVERSITÁRIA
}

\author{
BLOOD PRESSURE VALUES BETWEEN WORKERS \\ OF A UNIVERSITY INSTITUTION
}

\author{
VALORES DE PRESION ARTERIAL EN TRABAJADORES \\ DE UNA INSTITUCION UNIVERSITARIA
}

\section{MARIA LÚCIA DO CARMO CRUZ ROBAZZI, EUGENIA VELLUDO VEIGA, MARIA SUELY NOGUEIRA, MIYEKO HAYASHIDA, MÁRCIA CARON RUFFINO ${ }^{1}$}

\begin{abstract}
RESUMO
Realizou-se estudo entre trabalhadores de uma faculdade de Enfermagem brasileira, para identificar seus valores da pressão arterial (PA). Efetuou-se um levantamento dos registros de prontuários de consultas realizadas com estas pessoas, cadastradas em um programa de atendimento de PA da faculdade. De 149 trabalhadores, 51 constituíram a amostra. Os valores pressóricos foram medidos de modo padronizado e registrados nos prontuários durante 18 meses. Estas 51 pessoas tiveram 405 mensurações de PA, considerando-se para tal estudo apenas as medidas ocorridas com os sujeitos em posição sentada. Entre todas as categorias ocupacionais estudadas, os Vigias Prediais, os Operadores de Máquinas Reprográficas e os Motoristas foram os trabalhadores que apresentaram os maiores valores médios de PA, resultados coincidentes com a literatura consultada. Além disso, cinco trabalhadores estavam hipertensos, sendo que um desconhecia esta situação. Os achados evidenciam a necessidade de aprofundar investigações específicas relacionadas às categorias ocupacionais, particularmente as pertencentes aos ambientes universitários.
\end{abstract}

Palavras chaves: Pressão arterial em adulto, Medida da pressão arterial, Hipertensão arterial, Trabalhadores, Ambiente universitário.

\begin{abstract}
The present study was developed among workers from a Brazilian College of Nursing with the purpose of identifying the values of their blood pressure (BP). Data were collected from the records of these workers registered in a BP Program at the Unit. Among the 149 workers, the sample was formed by 51 of them. The values were measured in a standardized way and registered in records during 18 months. There were 405 measurements in the sitting position. Authors found out that among the occupational categories that were studied, the guards, Xerox machine operators and drivers had higher BP levels and these results are similar to the ones found in the literature. Also, 5 workers were hypertensive and one of them did not know about this situation. Findings showed the need to develop more specific research related to occupational categories, especially the ones from university fields.
\end{abstract}

Keywords: Blood pressure of adults, Measurement of blood pressure, Arterial hypertension, University workers.

\section{RESUMEN}

Fue realizado un estudio con los trabajadores de una Facultad de Enfermería del Brasil, para identificar valores de presión arterial (PA). Los investigadores realizaron una revisión de historias clínicas durante 18 meses. Los 51 trabajadores investigados presentaron 405 mediciones de PA, considerándose para el estudio, solamente aquellas

${ }^{1}$ Professoras da Escola de Enfermagem de Ribeirão Preto da Universidade de São Paulo, Centro Colaborador para o Desenvolvimento da Pesquisa em Enfermagem da Organização Mundial da Saúde/Organização Panamericana da Saúde. Avenida Bandeirantes n. 3900. CEP: 14040-902. Ribeirão Preto, São Paulo, Brasil. e-mail da primeira autora: avrmlccr@glete.eerp.usp.br 
realizadas con los sujetos en posición sentada. Entre todas las categorías ocupacionales estudiadas, los vigilantes de los predios, los operadores de máquinas reprográficas y los choferes fueron los trabajadores que presentaron altos valores medios de PA, resultados coincidentes con la literatura consultada. Asimismo, cinco trabajadores estaban hipertensos, uno de ellos desconocía esta situación. Los resultados evidencian la necesidad de profundizar investigaciones específicas relacionadas a categorías ocupacionales, particularmente en los ambientes universitarios.

Palabras claves: Presión arterial en adulto, Medida de la presión arterial, Hipertensión arterial, Trabajadores, Ambiente universitário.

\section{INTRODUÇÃO}

Saúde precária é condição relativamente freqüente entre os trabalhadores, em toda a sua história. No Brasil, as insatisfatórias condições laborais que lhes são impostas por grande número de empresas; as cargas excessivas de trabalho; o medo de desemprego; os baixos salários; os ritmos provocados pela maneira como é organizado o trabalho visando o aumento da produção; a falta de treinamento prévio; o descaso com os exames de saúde que devem ser realizados ou mesmo a total ausência de exames pré-admissionais ou periódicos; a não contratação de técnicos qualificados na área de segurança e saúde do trabalho; a não adesão e a falta de equipamentos de proteção individual; a ausência dos equipamentos de proteção coletiva, constituem-se apenas em alguns dos aspectos que favorecem aos trabalhadores a ocorrência de alterações à saúde. Em conseqüência, elevam-se os números de afastamentos, perdem-se vários dias de trabalho e em última instância, todo esse processo reverte em ônus para a empresa e para os próprios trabalhadores.

Há pessoas enfermas em decorrência do trabalho e de ambientes laborais nocivos; entretanto no Brasil, os verdadeiros números que revelam tal situação, não se mostram, em toda a sua plenitude, nos registros oficiais. A sub-estimação relacionada aos acidentes de trabalho e as doenças profissionais tem sido alvo de alertas $(18,19,200,206,21,27)$. Além dessas, há aquelas enfermidades relacionadas ao trabalho, em que o mesmo acaba sendo o fator contributo para o seu aparecimento ou agravamento ${ }^{(20 b, 21)}$. Complica-se esta situação quando se percebe que a comunidade trabalhadora brasileira acaba sendo proveniente de uma população também pouco saudável. Muitas pessoas ainda não têm acesso a moradia, transporte, estudo, adequado atendimento à saúde, lazer, trabalho compatível com um salário digno, bem como a uma alimentação básica, não garantindo em conseqüência, um padrão de saúde razoável. Considerando-se que estar com saúde é uma situação dinâmica, alternam-se os estados de mais ou menos saúde, em decorrência de problemas diversos, entre os quais, os já mencionados.

A Hipertensão Arterial (HA) é uma preocupante condição clínica multifatorial encontrada no mundo moderno, que pode ter o seu desenvolvimento favorecido devido a fatores de risco, como a hereditariedade, $\mathrm{o}$ sexo, a raça, a idade, o álcool, o fumo, o excesso de colesterol, a obesidade, o sedentarismo e os hábitos alimentares. Há que se considerar também o estresse, que além de outros fatores, pode estar presente no contexto do trabalho ${ }^{(15,32)}$.

Alguns estudos relacionaram a ocorrência de HA em grupos populacionais específicos ${ }^{(1,3,3,6,6,3,34,35,36) ;}$ não obstante, o país parece que ainda dispõe de poucas estatísticas que permitem estimar com certa segurança a sua freqüência e eventual impacto na saúde da população. A HA representa um dos grandes problemas de saúde pública, chegando a atingir quase 20 milhões de brasileiros, afetando muitos indivíduos em faixa etária produtiva ${ }^{(24)}$. Sua elevada prevalência implica em graus variáveis de incapacidade e diminuição na expectativa de vida do hipertenso, em virtude de doenças tais como insuficiên- 
cia cardíaca, acidente vascular cerebral, diabetes mellitus, doença coronariana e alterações renais ${ }^{(15,20 b)}$.

Sua prevenção, detecção e controle constituem-se então em um desafio para organismos governamentais, setores privados, profissionais de saúde, empregadores e empregados.

No Brasil, sabe-se que ainda há uma baixa cobertura no atendimento à população que sofre com tal problema, havendo necessidade de uma participação mais efetiva da equipe de saúde ${ }^{(15)}$. Os períodos de trabalho perdidos em sua decorrência têm sido expressivos, acarretando graves prejuízos, tanto para empresários como para trabalhadores e em última instância para a própria nação. Confirmam essa situação os dados da Seguridade Social ${ }^{(22)}$, os quais indicam ser a HA a terceira causa mais freqüente de auxílio doença e a primeira de aposentadoria por invalidez permanente no pais. Assim, detectá-la precocemente a fim de estabelecer o seu controle, com a realização de programas preventivos estruturados nas empresas, pode ser uma alternativa viável, por parte da classe patronal aos seus empregados.

A respeito das possíveis relações existentes entre HA e trabalho, encontrou-se na literatura internacional algumas investigações. Há autores que analisaram respostas cardiovasculares ao estresse ocupacional e cafeína entre empregados de "telemarketing"(9); que estudaram os efeitos dos ambientes de trabalho sobre a pressão sangüínea, em organizações de Nova Iorque ${ }^{(30)}$; que investigaram a prevalência de HA entre trabalhadores industriais em Stara Zagora ${ }^{(14)}$. Encontrou-se pesquisa que apresentou valores de pressão arterial(PA)entre empregados de uma fábrica têxtil ${ }^{(23)}$; que estudou a HA em trabalhadores expostos a ruído ocupacional ${ }^{(32)}$; que analisou os efeitos do estresse ocupacional na pressão sangüínea e levantou os riscos de HA entre mineiros ${ }^{(13,38)}$. Parece haver também incapacidade temporária ao trabalho determinada pela HA essencial ${ }^{(26)}$. Analisou-se a PA e a concentração sangüínea de chumbo em motoristas de ônibus de São Francisco ${ }^{(31)}$ e avaliou-se os fatores de risco para HA es- sencial em meio-ambiente industrial e esco$\operatorname{lar}^{(11)}$.

Em relato brasileiro, esta enfermidade foi associada particularmente como um dos efeitos ocasionados pelo ruído e pelo estresse ${ }^{(2)}$, que parece encontrar-se incluído em um grupo de "fatores psicológicos" ou "psicossociais"(20b). Considera-se que são várias as situações do ambiente laboral que podem ser estressoras, como carga de trabalho, insatisfação, alienação, monotonia e frustração, trabalho com rotatividade de turnos, conflitos interpessoais, competição, horários irregulares, trabalho noturno, oportunidades de promoção, entre outras. Encontrou-se diferença na prevalência de HA quando os trabalhadores foram agrupados segundo o ramo de atividade econômica e de classe social ${ }^{(20 b)}$, parecendo que a mesma ocorre mais em camadas socio-econômicas consideradas inferiores ou com diminuto grau educacional e em trabalhadores que executam tarefas mais diretamente relacionadas à produção ou atividades físicas pesadas, que às funções administrativas ou aquelas que permitem maior grau de autonomia, entre outras conclusões ${ }^{(2,20 b)}$.

Pode-se considerar então que no Brasil são relativamente poucas as investigações que mostram os valores de PA entre diferentes comunidades de trabalhadores. Sendo assim, estudos visando identificar aqueles com maior tendência à hipertensão, são recomendadas $^{(6)}$. Dessa forma pode-se tentar estabelecer quais são outros fatores que porventura tenham influência na elevação dos valores pressóricos e consequentemente encontrar esforços para sua eliminação. Elaborou-se então esse estudo, com o objetivo de identificar valores de PA entre trabalhadores de um instituição universitária.

\section{MATERIAL E MÉTODOS}

Realizou-se um levantamento nos registros dos prontuários de trabalhadores consultados em uma escola de enfermagem, pertencente à uma universidade pública, que tem entre os seus objetivos promover o ensino de 
graduação e pós-graduação, a pesquisa e a extensão de serviços à comunidade.

Os trabalhadores, todos da universidade, são cadastrados em um programa de atendimento de pressão arterial (PAPA) da faculdade; tal programa foi instituído pelos autores do presente estudo: a adesão ao mesmo, por parte dos trabalhadores, é voluntária.

De uma população de 149 trabalhadores, tanto professores como funcionários não docentes de diversas categorias ocupacionais, 51 (34,22\% do total) participaram do PAPA durante o período de 18 meses, ou mais precisamente, de janeiro de 1995 a junho de 1996.

Mediante agendamento prévio, o sujeito ausentava-se do seu turno, em média $20 \mathrm{mi}-$ nutos. O procedimento de medida dos valores pressóricos era realizado em uma sala de atendimento restrita, onde ele permanecia cinco minutos em repouso, antes de cada verificação, enquanto era orientado ao relaxamento físico e mental.

Os valores da PA eram obtidos através de medida indireta, utilizando-se esfigmomanômetro aneróide com manguito de $12 \mathrm{~cm}$ de largura e estetoscópio tipo "Tycos". Padronizou-se a verificação no membro superior direito, posicionado ao nível do coração, com a pessoa sentada e depois deitada e em posição supina, sendo realizadas duas medidas em cada uma destas posições. Para fins do presente estudo, os valores considerados foram os obtidos durante a segunda verificação, considerando-se que os níveis de PA variam e oscilam, durante o dia, em decorrência de vários fatores, incluindo-se a posição e o tônus muscular ${ }^{(15)}$. As informações foram registradas em formulários apropriados e posteriormente arquivadas em prontuários individuais.

Ressalta-se que a ocasião da coleta de dados do presente estudo foi anterior à Resolução 196/1996 do Conselho Nacional de Saúde ${ }^{(5)}$, divulgada em outubro daquele ano. No entanto, foram obedecidas as questões éticas vigentes no país, que até então referiamse à Declaração de Helsinque de 1964 e suas versões posteriores de 1975, 1983 e $1989^{(33)}$.
Durante o período de 18 meses anteriormente mencionado, foram realizadas nestas pessoas um total de 405 mensurações da PA nas três posições descritas anteriormente, considerando-se que os sujeitos retornavam ao atendimento mais de uma vez, agendados de modo prévio ou retornando espontaneamente. Desta forma houve uma média de 7.94 medidas por pessoa, no período em questão. Essas informações encontram-se visualizadas na tabela a seguir.

Tabela 1. Distribuição do número de verificações de PA segundo as posições supina, deitada e sentada de 51 trabalhadores cadastrados no PAPA, no período de 18 meses. Ribeirão preto (SP, Brasil), 1995-1996.

\begin{tabular}{|lcc|}
\hline Posição & \multicolumn{2}{c|}{ Mensuração da PA } \\
& $\mathrm{n}$ & $\%$ \\
\hline Supina & 98 & 24,2 \\
Deitada & 100 & 24,7 \\
Sentada & 207 & 51,1 \\
Total & 405 & 100,0 \\
\hline
\end{tabular}

Para a concretização do presente estudo, selecionou-se os valores obtidos com os trabalhadores na posição sentada por corresponderem a mais da metade das verificações realizadas no período.

\section{RESULTADOS E DISCUSSÃO}

Dos 51 sujeitos, $34(66,7 \%)$ eram do sexo feminino, $17(33,3)$ do masculino e tinham idade compreendida entre 19 e 55 anos, distribuídos nas seguintes faixas etárias: 24 (47,0\%) na de 19 a 30 anos; $14(27,4 \%)$ na de 31 a 40 anos e $13(25,5 \%)$ na de 41 a 55 anos. Houve predomínio de indivíduos de cor branca, com 42 pessoas $(82,3 \%)$ em relação aos $9(17,6 \%)$ não brancos.

De acordo com o Plano Universitário de Classificação de Cargos (PUCC), os trabalhadores não-docentes são enquadrados conforme as suas funções em Técnicos Operacionais (TO), Técnicos Especializados (TE) e Técnicos Administrativos (TA). Obedecendo essa classificação, os sujeitos desse estudo, exclu- 
indo-se os professores, foram divididos em algumas categorias ocupacionais.

Pertencentes a TO encontrou-se entre os atendidos os seguintes trabalhadores: $9.8 \%$ Ajudantes de Serviços Gerais (ASG), 5.9\% Operadores de Máquinas Reprográficas (OMR), 3.9\% Motoristas (M), 3.9\% Telefonistas (T) e 3.9\% Vigias Prediais (VP). Enquadrados nas funções TE foram atendidos: 3.9\% Técnicos de Ensino Médio em Ciências Biológicas e da Saúde (TEMCBS) e igual porcentagem de Técnicos de Ensino Médio em Produção e Análise de Dados (TEMPAD). Os trabalhadores classificados em TA eram secretários; também compuseram esta categoria os escriturários e os encarregados de compras e finanças, que foram agrupados genericamente como pertencentes aos SA - Serviços Administrativos - (54,6\%). 9,8\% dos que fizeram parte do estudo eram professores universitários (PU).

Os valores médios de pressão arterial sistólica (PAS) e diastólica (PAD), em milímetros de mercúrio ( $\mathrm{mmHg}$ ) entre essas categorias ocupacionais encontram-se demonstradas a seguir.

Tabela 2. Distribuição de 51 trabalhadores cadastrados no PAPA segundo as diversas funções e categorias ocupacionais e os valores médios de PAS e PAD, em mmHg, em mensurações realizadas nas posições sentada, no período de 18 meses. Ribeirão preto (SP, Brasil), 1995-1996.

\begin{tabular}{|ccccc|}
\hline Função & Categorias ocupacionais & $\mathrm{N}^{\circ}$ & PAS & PAD \\
\hline \multirow{4}{*}{ TO } & ASG & 14 & 115,9 & 73,1 \\
& OMR & & 131,7 & 70,3 \\
& M & & 120,0 & 90,0 \\
T & & 100,0 & 68,1 \\
TE & VP & 04 & 128,3 & 85,3 \\
& TEMCBS & 106,9 & 60,3 \\
TA & TEMPAD & 28 & 107,4 & 65,4 \\
& SA & 28 & 113,1 & 70,3 \\
& PU & 05 & 104,1 & 68,6 \\
\hline
\end{tabular}

Fuente: Valores de pressão em trabalhadores...

Os maiores valores médios de PAS e PAD encontram-se entre os OMR (PAS $=131,7$ $\mathrm{mmHg}$ e $\mathrm{PAD}=70,3 \mathrm{mmHg}), \mathbf{M}(\mathrm{PAS}=120$ $\mathrm{mmHg}$ e $\mathrm{PAD}=90 \mathrm{mmHg}$ ) e VP $(\mathrm{PAS}=128,3$ $\mathrm{mmHg}$ e $\mathrm{PAD}=85,3 \mathrm{mmHg})$. Sabe-se que 140,0 por $90,0 \mathrm{mmHg}$ (PAS por PAD) são considerados valores de normalidade para PA ${ }^{(29,34,35)}$.

Apesar da reduzida casuística apresentada nesse estudo e de terem sido encontrados, em poucas das categorias estudadas, isoladamente, valores de PA que possam ser considerados elevados, acredita-se que a ocorrência de maiores valores médios entre VP, OMR e $\mathbf{M}$ possa estar, de certa forma, relacionada às tarefas características que realizam tais pessoas durante o seu turno de trabalho na faculdade.
De acordo com o PUCC, os Vigias Prediais devem realizar as rondas de inspeção interna aos edifícios; adotar providências para evitar incêndios, roubos e danificações; fiscalizar as entradas e saídas de pessoas; zelar pela segurança nos locais e áreas sob sua inspeção; investigar e tomar as providências necessárias para quaisquer fatos anormais; atender ao público, identificando-o, entre outras tarefas. O horário laboral é feito em sistema de rodízio e eles costumam permanecer sozinhos em várias alas do prédio, principalmente durante a noite. Andam desarmados, tendo como única segurança o fato de trancarem as portas de acessos, a fim de protegerem o patrimônio e resguardarem-se de possíveis agressores externos. Estes trabalha- 
dores também são responsáveis pela guarda de equipamentos tanto grandes como pequenos, que podem ser retirados desapercebidamente, e, sendo assim, possivelmente essas situações podem ser estressoras e levam a pessoa a permanecer tensa, agravando consequentemente o risco de HA. Apesar de não se encontrar literatura disponível especificamente sobre esta ocupação e alterações dos valores de PA, estes trabalhadores realizam serviço de grande responsabilidade, possivelmente com tensão e sobrecarga psíquica, o que poderia ser uma das situações favorecedoras de estresse e consequentemente de elevação da PA.

Os Operadores de Máquinas Reprográficas realizam a tiragem de xerocópias de documentos requisitados; tiram provas dos trabalhos a fim de constatarem a qualidade de impressão; efetuam a manutenção preventiva ou mesmo pequenos reparos das máquinas reprográficas; controlam o recolhimento do numerário ou notas de débito, entre outras atribuições. As tarefas manuais que realizam são de operações repetitivas (colocar e retirar papéis das máquinas, espiralar teses e livros, grampear os textos reproduzidos, colocar-lhes capas, entre outras) ao mesmo tempo que efetuam atendimento ao público em espaço físico constituído por duas salas diminutas, local esse com ruído provocado pelo maquinário, pelas pessoas que permanecem nas áreas contíguas, pelo aparelho de refrigeração de ar e pelo telefone que soa constantemente, entre outros. Além disso, mantém contato permanente com as tintas e diluentes usados nas operações de impressão, que em geral têm como matéria prima o benzeno. A respeito desse agente químico, sabe-se que é um dos que mais pode provocar intoxicações profissionais ${ }^{(37)}$, apesar de ser desconhecida citação bibliográfica relacionando-o à modificações nos valores pressóricos. Como as máquinas são grandes, resta pouco espaço para os trabalhadores locomoverem-se no seu ambiente de trabalho, tendo eles que realizar a maior parte de seu trabalho em pé. Locais com área física pequena, em que as pessoas permanecem em ambientes ruidosos e realizam trabalho que exige prontidão no atendimento ao público e alerta em relação ao maquinário, provavelmente fazem os trabalhadores sentirem-se pressionados, fato esse que pode contribuir para a apresentação de valores de PA mais elevados. A exemplo da situação anterior, igualmente não se encontrou na literatura disponível estudos sobre este tipo de trabalhador, no entanto evidenciou-se investigação que descobriu maiores níveis pressóricos entre trabalhadores que realizam seu trabalho na posição pé, quando comparados aos que trabalham sentados ${ }^{(10)}$.

Em número reduzido, os Motoristas realizam habitualmente horas-extras, principalmente quando fazem viagens à outras cidades, à serviço da instituição. Dentre as suas atribuições estão dirigir automotores para transporte individual ou coletivo de passageiros e cargas de modo correto; inspecionar o veículo antes da partida; providenciar a manutenção do mesmo, especialmente em relação a lubrificação, lavagem, conservação pneumática, bateria, combustível; efetuar reparos de emergência durante o percurso, bem como a troca de pneus, correias e outros; mantê-lo em perfeito estado de funcionamento, entre outras. Os horários de trabalho tornam-se imprecisos, necessitando muitas vezes prolongarem o turno laboral até a noite ou mesmo madrugada. Além de transportarem pessoas, buscam equipamentos valiosos para a instituição, correndo o risco de assaltos ao veículo, durante os percursos; submetem-se também ao risco freqüente de acidentarem-se em episódios decorrentes do trânsito. Considera-se então que essa é uma das ocupações que mais risco apresenta, pelas variadas condições e situações agressivas, às quais os indivíduos são submetidos; podendo também contribuir para isso o rol de responsabilidades apontadas e os riscos aos quais os sujeitos são quotidianamente submetidos, que os podem levar a apresentar valores mais elevados de PA.

Apesar de não se encontrar literatura específica sobre o trabalho de motoristas de universidades e sua relação com a HA, en- 
controu-se alguns estudos sobre os motoristas de ônibus urbanos nacionais e de outros países, que constituem uma categoria ocupacional mencionada como possuindo elevada prevalência de $\mathrm{HA}^{(8 a, 8 b, 25,31)}$, em decorrência de instrumentos, ambientes e condições de trabalho dificultadas, apresentando situações estressoras relacionadas ao trânsito, condições de veículos, condições gerais de trabalho, entre outras. Como os trabalhos de ambos os tipos de motoristas acabam assemelhando-se, justifica-se os níveis pressóricos mais elevados na categoria $\mathbf{M}$.

Todas estas pessoas, coincidentemente, pertencem à ocupações consideradas socioeconomicamente menos favorecidas dentro do quadro funcional da universidade e, conseqüentemente, mais obedecedoras de ordens dentro do ambiente universitário; estudos nacionais têm assinalado que este quesito pode favorecer a ocorrência de HA $(2,20 b)$.

Do total de 51 sujeitos, cinco $(9,8 \%)$ tiveram os níveis de PA maiores ou iguais a 140 x $90 \mathrm{mmHg}$. Dentre eles quatro (8\%) sabiam que eram hipertensos e pertenciam as categorias de ASG e VP, o que pode explicar o maior número de pessoas com PA alterada entre os que exercem a função "menos qualificada", mais reprimida e de condições sociais e econômicas menos favorecida de TO. Um $(2 \%)$ da categoria SA desconhecia os seus valores pressóricos.

\section{CONCLUSÕES E RECOMENDAÇÕES}

Os resultados apresentados permitem concluir que:

-Os valores médios de PA encontrados entre os trabalhadores que exercem a função Técnicos Operacionais foram: $115,9 \mathrm{mmHg}$ para PAS e 73,1 mmHg para PAD entre os Ajudantes de Serviços Gerais; $131,7 \mathrm{mmHg}$ para PAS e 70,3 mmHg para PAD entre os Operadores de Máquinas Reprográficas; 120,0 $\mathrm{mmHg}$ para PAS e $90,0 \mathrm{mmHg}$ para PAD entre os Motoristas; 100,0 $\mathrm{mmHg}$ para PAS e 68,1 mmHg para PAD entre os Telefonistas e $128,3 \mathrm{mmHg}$ para PAS e $85,3 \mathrm{mmHg}$ para PAD entre os Vigias Prediais;

-Entre os que exercem a função Técnicos Especializados, os valores médios de PA encontrados foram: $106,9 \mathrm{mmHg}$ para PAS e $60,3 \mathrm{mmHg}$ para PAD entre os Técnicos de Ensino Médio em Ciências Biológicas e da Saúde e 107,4 mmHg para PAS e 65,4 mmHg para PAD entre os Técnicos de Ensino Médio em Produção e Análise de Dados;

-Quanto aos Técnicos Administrativos, os valores médios de PA encontrados entre os trabalhadores dos Serviços Administrativos foram de 113,1 para PAS e 70,3 para PAD; entre os Professores Universitários foram de 104,1 para PAS e 68,6 para PAD;

-As categorias ocupacionais que apresentaram os valores médios de PA mais elevados foram as Operadores de Máquinas Reprográficas, Motoristas e Vigias Prediais, que coincidentemente enquadram-se no grupo de trabalhadores socio-economicamente menos favorecidos da universidade e que mais ordens recebem da instituição, quesito esse que parece encontrar-se relacionado a elevação de valores de PA, de acordo com a literatura consultada;

-Do total de indivíduos estudados, cinco $(9,8 \%)$ apresentaram valores pressóricos no limite ou maiores que os preconizados pela Organização Panamericana de la Salud (24); desses a maioria pertencia às categorias que exerciam a função Técnico Operacional e conhecia o fato de possuir tais valores alterados.

Em decorrência da pequena casuística do presente estudo e da ainda pouca literatura existente sobre o assunto, torna-se difícil fazer associação entre valores de PA alterados e categorias ocupacionais. No entanto, os achados certamente evidenciam, a necessidade de aprofundar investigações específicas relacionadas às categorias ocupacionais pertencentes a ambientes universitários e valores pressóricos, contando com casuística maiores, que consigam oferecer mais subsídios para se perceber alterações de valores de PA e distintas categorias de trabalhadores. 


\section{REFERÊNCIAS BIBLIOGRÁFICAS}

1. ALMEIDA, D. B. et al. Estudo comparativo da pressão arterial e da prevalência de hipertensão arterial em dois coortes sucessivos (1975-1976) de estudantes de 16 a 25 anos, Botucatú, SP, Brasil. Rev.Saúde públ., São Paulo, Brasil, 12, pp. 497-505, 1978.

2. ANDRADE Filho, A; SANTOS Júnior, E. A. Aparelho cardiovascular. In: MENDES, R. Patologia do trabalho. Rio de Janeiro, Brasil, Ed. Atheneu, 1995. pp. 311-28.

3. ARCURI, E. A. M. Estudo comparativo da medida indireta da pressão arterial com manguito de largura correta e com manguito de largura padrão. São Paulo, 1985. Tese (Doutorado) - Instituto de Ciências Biomédicas, Universidade de São Paulo.

4. BARRETTO, M.L.; MEIRA, R. L. C. Hipertensão arterial em uma comunidade do oeste da Bahia (Brasil) Arq.Bras.Cardiol., São Paulo, Brasil, 34 (5), pp. 363-6, 1980.

5. BRASIL. Ministério da Saúde. Conselho Nacional de Saúde. Resolução 196 de 10 de outubro de 1996. Brasília, Brasil, 1996.

6. CARVALHO, J. J. M. et al. Pressão arterial e grupos sociais. Estudo epidemiológico. Arq.Bras.Cardiol. São Paulo, Brasil, 40 (2), pp. 115-20, 1983.

7. CHIAVERINI, R. et al. Hipertensão arterial para o clínico. Rio de Janeiro, Brasil, Ed. Atheneu, 1985.

8a. CORDEIRO, R.; LIMA Filho, E. Impacto do trabalho sobre a pressão arterial entre trabalhadores jovens e idosos do setor de transporte da cidade de Campinas. In: Congresso Brasileiro de Epidemiologia, $2^{\circ}$, Belo Horizonte, 1992. Anais. Rio de Janeiro, Brasil, Abrasco, 1992. p. 120.

8b. CORDEIRO, R.; LIMA Filho, E. Pressão arterial entre motoristas e cobradores de Campinas. In: Congresso Brasileiro de Epidemiologia, 2, Belo Horizonte, 1992. Ana ais. Rio de Janeiro, Brasil, Abrasco, 1992. p. 120.

9. FRANCE, C.; DITTO, B. Cardiovascular responses to occupational stress and caffeine in telemarketing employees. Psychosom.Med., 51, (2), pp. 145-51, 1989.

10. FOURIAUD, C.et al. Influence of socioprofessional conditions on blood pressure levels and hypertension control. Am J.Epidemiol., (120), pp. 72-86, 1984.

11. IVAN, A. et al. Evaluation of risk factors for essencial arterial hypertension in industrial and school environments. Santé Publique, 33, (1), p.39-48, 1990.

12. KOCHAR, M. S.; WOODS, K. D. Controle da hipertensão para enfermeiros e demais profissionais de saúde. São Paulo, Brasil, Ed. Andrei, 1990.

13. KOVALENKO, V. N. et al. Faktory riska gipertonicheskoi bolezni u gornorabochikh zhelezorudnykh shakht. Sov.Zdravookhr, (12), pp. 30-3. 1989
14. KURNOLSKI, I. et al. Epidemiologichni prouchvaniia vurkhu razprostranenieto na arterialnata khipertoniia tri promishleni rabotnitsiv Stara Zagora. Vutr.Boles, 28 (3). pp. 4852. 1989.

15. LIMA, F.E.T. et al. Conhecimento dos auxiliares e técnicos de enfermagem quanto aos fatores relacionados à pressão arterial. Nursing, São Paulo, 3 (24): pp. 18-23. 2000.

16. LUNA, R. L. Hipertensão Arterial. São Paulo, Medsi, 1989. 301 p.

17. MEIRA, A. R. Saúde e doença. In: PARETA, J. M. M. et al. . Saúde da Comunidade, São Paulo, Brasil, Ed. Mc Graw-Hill do Brasil, 1976. cap. 1, pp. 15 .

18. MEDRADO-FARIA, M. A. Saúde e trabalho industrial: condições de saúde dos operários brasileiros. Ciência e Cultura, 40 (10), pp. 967-75. 1988.

19. MENDES, R. Doutrina e prática de integração da saúde ocupacional no setor saúde: contribuição para a definição de uma política. São Paulo, 1986. Tese (Livre-Docência) - Faculdade de Saúde Pública, Universidade de São Paulo.

20a. MENDES, R. Subsídios para um debate em torno da revisão do atual modelo de organização ocupacional no Brasil.Rev.Bras.Saúde Ocup., São Paulo, Brasil, 64 (16), pp. 7-25. 1988.

20b.MENDES, R. O impacto dos efeitos da ocupação sobre a saúde dos trabalhadores: 1 . Morbidade.

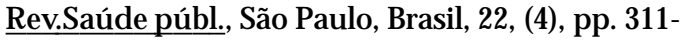
26. 1988.

21. MENDES, R. Aspectos conceituais da patologia do trabalho. In: MENDES, R. Patologia do trabalho. Rio de Janeiro, Brasil, Ed. Atheneu, 1995, pp. 3347.

22. MINISTÉRIO DA SAÚDE - Normas, Técnicas para o Programa Nacional de Educação e Controle da Hipertensão Arterial (PNECHA). Centro de Documentação do Ministério da Saúde, Brasileira, 1988.

23. MLADENOVSKI, B. Prouchvaniia vurkhu arterialnoto naliagane na rabotnitsite i sluzhitelite v edno tekstilno predpriiatie. Vutr.Boles, 29 (2), pp. 63-9. 1990.

24. ORGANIZAÇÃO PANAMERICANA DE LA SALUD - La hipertension arterial como problema

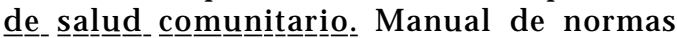
operativas para um programa de controle en los diferentes niveles de atención, N ${ }^{\circ} 3.1990$.

25. PINHO, C. et al. Alterações cardiovasculares em motoristas de ônibus. Rev.Bras.Saúde Ocup., São Paulo, Brasil, (19), pp. 53-8. 1991.

26. POLL, N. Considerations sur líncapacite temporaire de travail, determinee par l'hypertension arterielle essentielle.Santé Publique, 31 (3), pp. 225-30. 1988.

27. POSSAS,C.A . Avaliação da situação atual do sis- 
tema de informação sobre doenças e acidentes do trabalho no âmbito da previdência social brasileira e propostas para sua reformulação. Rev.Bras.Saúde Ocup., São Paulo, Brasil, 60, (15), pp. 43-67. 1987.

28. REED. D. M. et al. Occupational strain and the incidence of coronary heart disease. A․․ I. Epidemiol., 129, (3), pp. 495-502. 1989.

29. RIBEIRO, A .B. Hipertensão arterial. Rio de Janeiro, Brasil. Editora Marques Saraiva, 1988.

30. SCHLUSSEL, Y. R. et al. The effect of work environments on blood pressure: evidence from seven New York organizations. J.Hypertens. 8, (7), pp. 679-85. 1990.

31. SHARP, D. S. et al.. Blood pressure and blood lead

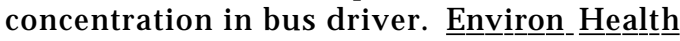
Perspect., (78), pp. 131-7. 1988.

32. TALIJANCIC, A.; MUSTAC, M. Arterijska hipertenzija u radnika profesionalno izlozenih buci. Arh Hig Rada Toksikol, 40, (4), pp. 415-20. 1989.

33. UNIVERSIDADE DE SÃO PAULO. Escola de Enfermagem de Ribeirão Preto. Comitê de Ética em Pesquisa da Escola de Enfermagem de Ribeirão Preto. Diretrizes e normas regulamentadoras de pesquisa envolvendo seres humanos. 44 pp. 1999.
34. VEIGA, E. V. Pressão arterial em escolares: um estudo de medidas e variabilidade. Ribeirão Preto, 1987. Dissertação (Mestrado) - Escola de Enfermagem de Ribeirão Preto,Universidade de São Paulo.

35. VEIGA, E. V.et al. Estudo dos fatores de risco da hipertensão arterial: conhecimento e exposição. Rev Soc Cardiol Estado de São Paulo, 3, (6, supl. A). 1993.

36. VINHA,V.H.P. Estudo da pressão arterial em policiais militares do grupo etário 20-50 anos. Ribeirão Preto, 1972, Tese. (Doutorado). Escola de Enfermagem de Ribeirão Preto, Universidade de São Paulo.

37. WAKAMATSU, C. T.; FERNÍCOLA, N. A. G. G Intoxicação profissional por benzeno. In: MENDES, R. Medicina do trabalho. Doenças profissionais. São Paulo, Brasil, Ed. Sarvier, 1980. pp. 479-88.

38. ZWINGENBERGER, W. et al. Einfluss der arbeitsbelastung auf das blutdruckverhalten von bergarbeitern im erzbergbau. Z Gesamte Hyg., 35 (5), pp. 269-71. 1989. 